\title{
Phase I and Pharmacologic Study of Oral ZD9331, A Novel Nonpolyglutamated Thymidylate Synthase Inhibitor, in Adult Patients With Solid Tumors
}

\author{
By Maja J.A. de Jonge, Cornelis J.A. Punt, Alex Sparreboom, André S.T. Planting, M.E.W.J. Peters, \\ Jacqueline van de Schraaf, Ann Jackman, Rob Smith, Pieter H.M. de Mulder, and Jaap Verweij
}

\begin{abstract}
Purpose: To assess the toxicity profile and doselimiting toxicities (DLTs), to determine the maximumtolerated dose, and to study the pharmacokinetics of ZD9331 when administered orally to patients with advanced solid tumors.

Patients and Methods: Patients were treated with oral ZD9331 given once daily (od) or twice daily (bid) for 5, 7, or 10 days; cycles were repeated every 21 days at doses ranging from $\mathbf{2 . 5}$ to $\mathbf{4 0} \mathrm{mg}$. For pharmacokinetic analysis, plasma sampling was performed during the first course and assayed using a validated liquid chromatographic-tandem mass spectrometry assay. Plasma levels of 2'-deoxyuridine were measured as a surrogate marker for TS inhibition.

Results: Forty-two patients received a total of 166 courses. The DLTs were myelosuppression and skin
\end{abstract}

rash. Dose escalation of oral ZD9331 from 2.5 to 40 $\mathrm{mg}$, as a single daily dose, resulted in a less than proportional increase in the plasma area under the concentration-time curve of ZD9331. The plasma drug exposure per cycle for the schedules $20 \mathrm{mg}$ od for 5 days, $10 \mathrm{mg}$ od for 10 days, and $10 \mathrm{mg}$ bid for 5 days, all resulting in a total dose per cycle of $100 \mathrm{mg}$, were comparable. One partial response was noted in a patient with gastric cancer.

Conclusion: DLTs in this phase I study of oral ZD933 I were myelosuppression and skin toxicity. The recommended dose for phase II studies of oral ZD9331 is 20 mg od for 5 consecutive days, every 3 weeks.

J Clin Oncol 20:1923-1931. () 2002 by American Society of Clinical Oncology.

In vitro studies have shown that ZD9331 is a potent inhibitor of $\mathrm{TS}\left(\mathrm{K}_{\mathrm{i}}=0.4 \sim \mathrm{nmol} / \mathrm{L}\right),{ }^{13}$ and antitumor activity has been documented in vivo against human ovarian, colon, non-small-cell lung, and gastric carcinoma xenograft models. ${ }^{14}$ In addition, antitumor activity has been observed after intravenous administration of ZD9331 in patients with a variety of solid tumors. ${ }^{15-18}$ As expected in view of the lack of intracellular drug retention of ZD9331, the requirement for prolonged drug exposure to inhibit TS and exert an antitumor effect suggested that ZD9331 might be more active when given by a continuous infusion. Alternatively, prolonged exposure might also be achieved in a possibly more patient-convenient way, ie, oral administration of the drug. When given orally to rodents, ZD9331 retained its antitumor activity (data on file, AstraZeneca,

From the Department of Medical Oncology, Rotterdam Cancer Institute (Daniel den Hoed Kliniek) and University Hospital, Rotterdam, and Department of Medical Oncology, University Medical Center St Radboud Nijmegen, Nijmegen, the Netherlands; and Institute of Cancer Research, Surrey, and AstraZeneca, Alderley Park, United Kingdom.

Submitted May 11, 2001; accepted December 17, 2001.

Address reprint requests to M.J.A. de Jonge, MD, PhD, Department of Medical Oncology, Rotterdam Cancer Institute (Daniel den Hoed Kliniek) and University Hospital, Groene Hilledijk 301, 3075 EA Rotterdam, the Netherlands; email: jonge@onch.azr.nl.

(C) 2002 by American Society of Clinical Oncology.

0732-183X/02/2007-1923/\$20.00 


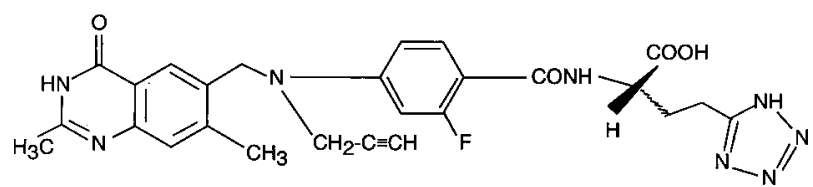

Fig 1. Chemical structure of ZD9331.

Alderley Park, United Kingdom). Toxicity observed in animals after oral administration of ZD9331 included weight loss, decreased food consumption, myelosuppression (especially leukocytopenia), and gastrointestinal toxicity (data on file, AstraZeneca).

The pharmacokinetics of oral ZD9331 have been studied in several animal species. Following oral administration of ZD9331 to dogs, the absorption was rapid, with peak plasma concentrations occurring after 0.25 to 2 hours. After single and daily oral doses of $0.05,0.1$, and $0.2 \mathrm{mg} / \mathrm{kg} / \mathrm{d}$, the area under the plasma concentration-time curve (AUC) of ZD9331 increased proportionally with dose, and bioavailability was approximately $80 \%$. At higher dose levels (1 $\mathrm{mg} / \mathrm{kg} / \mathrm{d}$ ), however, a less than dose-proportional increase in both the maximal concentration and AUC of ZD9331 was observed; a 25-fold increase in dose resulted in only a threeto four-fold increase in plasma exposure, suggesting a saturable absorption mechanism. Terminal half-life values for ZD9331 after oral administration ranged from 7 to 11 hours in rats and from 5 to 70 hours in dogs. Excretion of ZD9331 in both rat and dog was mainly via the bile as unchanged parent compound. Biliary recirculation of ZD9331 was apparent (data on file, AstraZeneca).

The objectives of this phase I study were to assess the toxicity profile of oral ZD9331, to determine the maximumtolerated dose, to document any antitumor effects, and to study the pharmacokinetics of ZD9331 administered orally to patients with advanced solid tumors.

\section{PATIENTS AND METHODS}

\section{Study Design}

This was an open, nonrandomized, noncomparative, dose-escalating trial of ZD9331 administered orally, once or twice daily, to adult patients with refractory solid tumors. The trial was designed to comply with the ethical principals of Good Clinical Practice in accordance with the Declaration of Helsinki. The study was approved by the institutions' medical ethics committees, and all patients had to give written informed consent before study entry.

\section{Patient Selection}

Patients with a histologically confirmed diagnosis of a malignant solid tumor refractory to standard forms of therapy were eligible. Other eligibility criteria included the following: age $\geq 18$ years; World Health Organization performance status $\leq 2$; estimated life expectancy more than 12 weeks; no previous anticancer therapy for at least 4 weeks (6 weeks for nitrosoureas or mitomycin); and adequate hematopoietic (absolute peripheral granulocyte count $\geq 2.0 \times 10^{9} / \mathrm{L}$ and platelet count $\geq 100 \times 10^{9} / \mathrm{L}$ ), hepatic (bilirubin $\leq 1.25$ times upper limit of normal $[\mathrm{ULN}]$ and serum AST and ALT $\leq 2.5$ times ULN or $\leq$ five times ULN in case of liver metastases), and renal (serum creatinine $\leq$ 1.25 times ULN) function. Concomitant administration of folic acid was not allowed.

\section{Treatment and Dose Escalation}

ZD9331 was supplied as film-coated tablets that contained 0.5, 2, 5, or $10 \mathrm{mg}$ of the active drug, and these were stored at $4^{\circ} \mathrm{C}$. The tablets were taken once daily (od) or twice daily (bid) with a glass of water at least 30 minutes before breakfast after an overnight fasting and, for bid dosing, before the evening meal after at least 2 hours of fasting. Patients were treated on an outpatient basis. The daily dose of ZD9331 was provided in separate pots, with each daily dosing clearly identifiable by the patient. Patients were instructed to record their daily amount of tablets taken, the time of administration, and the timing in relation to their meals. Compliance with the scheduled treatment was assessed at the end of each course, by counting the used and returned tablets of ZD9331 in relation to the record kept by the patient for the given cycle.

The starting dose of ZD9331, $2.5 \mathrm{mg}$ given orally od for 5 days, was one third of the minimal toxic dose in dogs. Courses were to be repeated every 21 days. Dose escalations were based on the prior dose level toxicity. If no toxicity (excluding alopecia, mild or moderate fatigue, grade 1 or 2 nausea and vomiting, and transient rise in AST and/or ALT) was observed at the previous dose level, a 100\% dosage increment was allowed. In this accelerated stage, at least one patient was treated at each dose level. However, if toxicity was observed, a dose escalation of $15 \%$ to $67 \%$ (determined by the worst significant toxicity) according to a modified Fibonacci scheme was prescribed. From that time onward, at least three patients were entered at each dose level. Once the dose-limiting toxicity (DLT) was observed in one out of three patients, three additional patients were treated at that dose level. Based on pharmacokinetic findings, the dosing scheme and the duration of drug administration could be amended during the study. The maximumtolerated dose was defined as one dose level below the dose that induced DLTs in more than $50 \%$ of patients during course 1. DLTs were defined according to the National Cancer Institute's common toxicity criteria (version December 1994) and comprised grade 4 neutropenia for at least 7 days, grade 4 neutropenic fever, grade 4 thrombocytopenia, and nonhematologic toxicity $\geq$ grade 3 not ameliorated by symptomatic measures (excluding a transient rise in AST and/or ALT). Intrapatient dose escalation was only allowed during the accelerated phase of dose escalation. If a patient encountered DLT, the dose of ZD9331 was decreased by one dose level at retreatment. The treatment was resumed when the neutrophil count had recovered to $\geq 2.0 \times 10^{9} / \mathrm{L}$, the platelet count to $\geq 100 \times 10^{9} / \mathrm{L}$, and all other redosing criteria were met.

\section{Treatment Assessment}

Before therapy, a complete medical history was taken and a physical examination was performed. A complete blood cell count including WBC differential and serum biochemistry, which involved sodium, potassium, calcium, phosphorus, urea, creatinine, total protein, albumin, total bilirubin, alkaline phosphatase, AST, ALT, gamma-glutamyl transferase, glucose, and uric acid, were performed, as were urinalysis, ECG, and a chest $\mathrm{x}$-ray. Weekly evaluations included history, physical examination, toxicity assessment according to the common toxicity criteria, and serum chemistry. The complete blood cell count was determined twice weekly. Tumor 
evaluation was performed after every two courses according to the World Health Organization criteria for response. Patients were taken off protocol at the onset of disease progression.

\section{Sample Collection and Drug Analysis}

For pharmacokinetic analysis, 23 to 29 blood samples $(\sim 3 \mathrm{~mL})$ were obtained from an indwelling intravenous cannula and collected in vials containing lithium heparin as anticoagulant. The samples were taken before dosing, at $1,2,4,6,8,12$, and 24 hours after dosing on days 1 and 4 , at 2 hours after dosing on day 2, before dosing on day 3 , and 2 hours after dosing on day 3 of the first course. Additional samples were taken $72,168,240$, and 408 hours after the last dosing on day 5. All samples were centrifuged immediately after sampling for 10 minutes at $1,000 \times g$, and the plasma supernatant was snap-frozen at $-20^{\circ} \mathrm{C}$ and remained frozen until analysis.

The protocol allowed changes in the administration scheme of ZD9331 based on pharmacokinetic data obtained during the study. Indeed, several other schemes were studied. This resulted in an adaptation of the pharmacokinetic sampling points.

In the 5-day bid regimen, blood samples were taken before the morning and evening doses of the drug, 1, 2, 4, 6, and 8 hours after the morning dose, and 1, 2, and 4 hours after the evening dose on days 1 and 4 (or 5) of the first cycle. Additional samples were taken before the morning and evening doses on days 2 and 3. During the 7-day od regimen, samples for pharmacokinetic analysis were taken on days 1 and 7 before dosing, at 1 , 2, 4, 6, and 12 hours after dosing, and before dosing and 2 hours after dosing on days 2,3 , and 6 . For the 10-day od regimen, samples were taken before dosing, at 1, 2, 4, 6, 12, and 24 hours after dosing on days 1 and 10, before dosing and 2 hours after dosing on days $2,3,4,7,8$, and 9, and 120, 192, and 288 hours after the last dosing on day 10. Additional samples were taken up to 288,360 , and 480 hours after the final dosing for the 10-, 7-, and 5-day regimens, respectively. Concentrations of ZD9331 were determined according to a validated liquid chromatographic-tandem mass spectrometry assay.

Inhibition of TS leads to a rise in the level of intracellular dUMP, which is phosphorylated to 2 'deoxyuridine (dUrd). dUrd is effluxed from cells, leading to a rise in plasma dUrd. ${ }^{19,20}$ Thus, plasma dUrd can be used as a surrogate marker for TS inhibition. ${ }^{21,22}$ Samples (nos. 7 to 11) for the determination of plasma dUrd $(\sim 8 \mathrm{~mL})$ were taken from dose level $10 \mathrm{mg}$ bid for 5 days onward and collected in vials containing lithium heparin as anticoagulant. The samples were obtained before dosing on days 1 to 5 , on days 8,15 , and 22 in the 5-day regimens, on days 1 to 3,6 to $8,10,14,17$, and 22 in the 7 -day regimen, and on days 1 to 4,7 to $11,15,18$, and 22 in the 10-day regimen. All samples were centrifuged immediately at $1,000 \times g$ for 10 minutes at $4{ }^{\circ} \mathrm{C}$, and the plasma supernatant was snap-frozen at $-20^{\circ} \mathrm{C}$ until high-performance liquid chromatographic analysis. Concentrations of plasma dUrd were determined according to a validated high-performance liquid chromatographic assay, which was performed as described previously, ${ }^{21}$ with fluorescence detection at $301 \mathrm{~nm}$.

\section{Pharmacokinetic and Pharmacodynamic Data Analysis}

The terminal disposition half-life of ZD9331 was calculated as $\ln 2 / k$, where $k$ is the terminal elimination rate constant (expressed in hours ${ }^{-1}$ ) following the last dose. The peak plasma concentrations and the time to peak plasma concentration were determined graphically from the (observed) experimental values. The AUC of ZD9331 was estimated using the experimental values (trapezoidal rule) with extrapolation to infinity using the terminal elimination rate constant, defined as the slope of at least the final three data points of the log-linear concentration-time plot.
Pharmacokinetic/pharmacodynamic relationships between ZD9331 kinetic parameters and hematologic toxicity associated with drug administration were evaluated using the Siphar software package (InnaPhase, Philadelphia, PA). Only the first course of each patient was taken into consideration to avoid potentially confounding bias due to cumulative toxicity. All data were fitted to a sigmoidal maximum effect model based on the modified Hill equation, as follows:

$$
\mathrm{E}=\mathrm{E}_{0}+\mathrm{E}_{\max } \times\left[\left(\mathrm{KP}^{\gamma}\right) /\left(\mathrm{KP}^{\gamma}+\mathrm{KP}_{50}^{\gamma}\right)\right] .
$$

In this equation, $E_{0}$ is the minimum reduction possible, $E_{\max }$ is the maximum response, fixed at 100 (continuous variables) or 4 (discrete variable), $\mathrm{KP}$ is the pharmacokinetic parameter of interest, $\mathrm{KP}_{50}$ is the value of the pharmacokinetic parameter predicted to result in half of the maximum response, and gamma $(\gamma)$ is the Hill constant describing the sigmoidicity of the curve.

\section{Statistical Analysis}

All pharmacokinetic parameters are reported as mean values \pm SD. The pharmacokinetic-pharmacodynamic models were evaluated for goodness of fit by minimization of sums of the squared residuals and by reduction of the estimated coefficient of variation for fitted parameters. Significance of these relationships was assessed by construction of contingency tables with subsequent $\chi^{2}$ analysis. The correlations between the individual AUC values and the administered dose level was analyzed by means of Spearman's correlation coefficient (rho) and a linear regression analysis. Variability in pharmacokinetic parameters between the various dose levels was evaluated by the Kruskal-Wallis statistic followed by Dunn's multiple comparison test. Interpatient variability in oral clearance of ZD9331 was correlated with patient characteristics, including body-surface area, sex, and age, using uni-

Table 1. Patient Characteristics

\begin{tabular}{|c|c|c|}
\hline Characteristic & & $\begin{array}{l}\text { No. of } \\
\text { Patients }\end{array}$ \\
\hline No. entered & & 42 \\
\hline No. assessable & & 42 \\
\hline \multicolumn{3}{|l|}{ Age, years } \\
\hline Median & 58 & \\
\hline Range & $23-74$ & \\
\hline \multicolumn{3}{|l|}{ Sex } \\
\hline Female & & 10 \\
\hline Male & & 32 \\
\hline \multicolumn{3}{|l|}{ WHO performance status } \\
\hline 0 & & 17 \\
\hline 1 & & 20 \\
\hline 2 & & 5 \\
\hline \multicolumn{3}{|l|}{ Tumor type } \\
\hline Colorectal & & 20 \\
\hline (A)CUP & & 4 \\
\hline Renal & & 3 \\
\hline (N)SCLC & & 3 \\
\hline Miscellaneous & & 12 \\
\hline \multicolumn{3}{|l|}{ Previous treatment } \\
\hline Chemotherapy & & 20 \\
\hline Chemotherapy and radiation & & 15 \\
\hline None & & 7 \\
\hline
\end{tabular}

Abbreviations: (A)CUP, (adeno)carcinoma of unknown primary; (N)SCLC, (non)small-cell lung cancer; WHO, World Health Organization. 
variate linear regression analysis. A similar analysis was performed to evaluate potential relationships between the total cumulative exposure $\left(\mathrm{AUC}_{0-504}\right)$ to ZD9331 and measures of renal function (serum creatinine values) and hepatic function (bilirubin, AST, ALT, and alkaline phosphatase). Probability values and adjusted $r^{2}$ values were calculated as a measurement for correlation. Statistical calculations were performed using the Number Cruncher Statistical System version 5.X (J.L. Hintze, East Kaysville, UT, 1992). Probability values of less than .05 were regarded as statistically significant.

\section{RESULTS}

A total of 42 patients were enrolled onto the study at two institutions. Patient characteristics are listed in Table 1. Thirty-nine patients were eligible. Three patients were ineligible based on marginally elevated serum bilirubin levels; however, all patients were taken into consideration for the assessment of toxicity and response. The majority of the patients were either asymptomatic or had only mild symptoms. The most common tumor type was colorectal cancer. The total number of assessable courses was 166, and the median number of courses per patient was two (range, one to 14). Dose levels studied were 2.5, 5, 10, 20, 30, and $40 \mathrm{mg}$ od for 5 days, $10 \mathrm{mg}$ bid for 5 days, $10 \mathrm{mg}$ od for 10 days, and $20 \mathrm{mg}$ od for 7 days (Table 2). All cycles were repeated once every 3 weeks. In one patient treated at $10 \mathrm{mg}$ od for 5 days, the dose of ZD9331 was escalated from the third cycle onward to $20 \mathrm{mg}$ for 5 days, as allowed by protocol. In two patients $(20 \mathrm{mg} / \mathrm{d}, 40 \mathrm{mg} / \mathrm{d})$ dose reductions were necessary because of toxicity. Treatment was discontinued in five patients because of side effects (hematologic toxicity in one patient, skin toxicity in three, and skin/hematologic toxicity in one).

\section{Tolerability}

At first, the dose of ZD9331 administered daily was escalated. At the dose level $10 \mathrm{mg}$ od for 5 days, one patient experienced grade 3 hyperbilirubinemia. In view of the fact that the patient concomitantly suffered from pneumonia and

Table 2. Dose Levels and Number of Patients Entered

\begin{tabular}{cccc}
\hline Dose $(\mathrm{mg} / \mathrm{d})$ & Days per Cycle & $\begin{array}{c}\text { Total Dose per Cycle } \\
(\mathrm{mg})\end{array}$ & No. of Patients \\
\hline 2.5 od & 5 & 12.5 & 2 \\
5 od & 5 & 25 & 1 \\
10 od & 5 & 50 & 1 \\
20 od & 5 & 100 & $12(1)^{*}$ \\
30 od & 5 & 150 & $3(1)^{*}$ \\
40 od & 5 & 200 & 6 \\
10 bid & 5 & 100 & $6(1)^{*}$ \\
10 od & 10 & 100 & 6 \\
20 od & 7 & 140 & 5 \\
\hline
\end{tabular}

*Number in parentheses indicates patient treated at this dose level after dose escalation or reduction. cardiac dysfunction, it was decided to consider the hyperbilirubinemia to be not drug-related. Dose escalation proceeded. At $40 \mathrm{mg}$ od for 5 days, DLT was first encountered and consisted of grade 4 neutro- and thrombocytopenia in one out of 6 patients. However, pharmacokinetic data, to be described in more detail below, revealed no increase in plasma exposure to ZD9331 with doses over $20 \mathrm{mg}$. In view of these findings, no further dose escalation was pursued and the following alternative regimens were studied: $10 \mathrm{mg}$ bid dosed for 5 days, $20 \mathrm{mg}$ od for 7 days, and prolonged administration of $10 \mathrm{mg}$ od for 10 days. At the $10-\mathrm{mg}$ bid dose level, two out of six patients experienced DLT consisting of a grade 3 rash; in one of the two patients, it was in conjunction with grade 4 neutro- and thrombocytopenia. Also, at the dose level of $20 \mathrm{mg}$ od for 7 days, three out of five patients developed a grade 3 rash, and in the regimen applying the 10-day dosing of $10 \mathrm{mg}$, one patient out of six developed a grade 3 rash and another patient had grade 4 thrombocytopenia. At this point it was decided to extend the cohort of patients treated with $20 \mathrm{mg}$ od for 5 days to 12

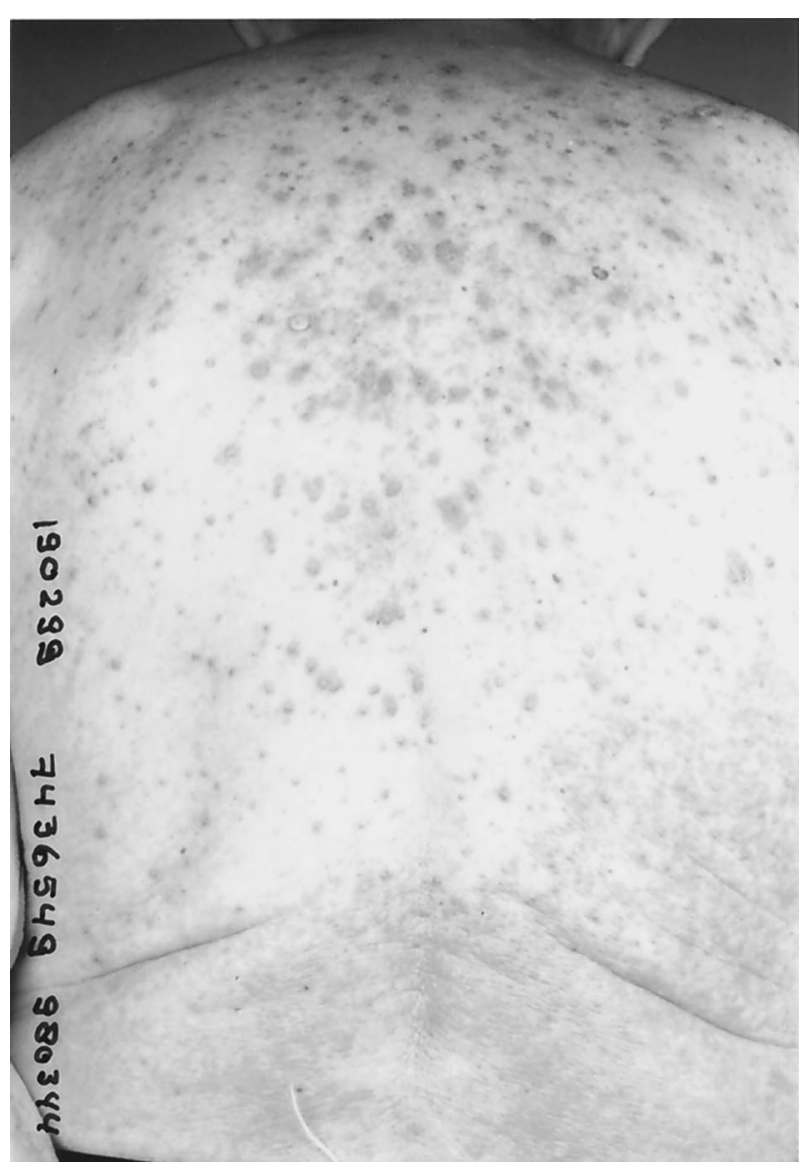

Fig 2. Typical example of grade 3 rash. 
Table 3. Worst Toxicity per Patient per Dose Level

\begin{tabular}{|c|c|c|c|c|c|c|c|c|c|c|}
\hline \multirow[b]{2}{*}{ Dose $(\mathrm{mg}) \times$ Days } & \multicolumn{2}{|c|}{ Neutropenia } & \multicolumn{2}{|c|}{ Thrombocytopenia } & \multicolumn{2}{|c|}{ Transaminases } & \multicolumn{2}{|c|}{ Skin } & \multicolumn{2}{|c|}{ Diarrhea } \\
\hline & Grade 3 & Grade 4 & Grade 3 & Grade 4 & Grade 3 & Grade 4 & Grade 3 & Grade 4 & Grade 3 & Grade 4 \\
\hline 2.5 od $\times 5$ & - & - & - & - & 1 & - & - & - & - & - \\
\hline 5 od $\times 5$ & - & - & - & - & - & - & - & - & - & - \\
\hline 10 od $\times 5$ & - & - & - & - & - & - & - & - & - & - \\
\hline 20 od $\times 5$ & - & - & - & - & 3 & - & 1 & - & 1 & - \\
\hline 30 od $\times 5$ & - & - & 1 & - & - & - & - & - & - & - \\
\hline 10 bid $\times 5$ & 1 & 1 & 1 & 1 & 2 & - & 2 & - & - & 1 \\
\hline 10 od $\times 10$ & - & - & - & 1 & - & - & 1 & - & - & - \\
\hline 20 od $\times 7$ & - & - & 1 & - & 2 & - & 3 & - & - & - \\
\hline
\end{tabular}

patients. One of the additional patients developed a grade 3 rash in conjunction with grade 3 diarrhea during the first treatment course, but no further DLTs were noted. In view of these data and the pharmacokinetic data, $20 \mathrm{mg}$ od for 5 days was considered the recommended dose for further study of this regimen.

Skin toxicity was the predominant nonhematologic toxicity observed over the total dose range studied and was encountered in $29 \%$ of the cycles and consisted of a rash predominantly located on the trunk and extremities (Fig 2). Microscopical examination of a skin biopsy revealed an infiltration with lymphocytes and eosinophils compatible with a toxic dermatitis. The median day of onset of the skin reaction was day 7 after the start of the treatment (range, days 1 to 17), with a median duration of 12 days (range, 1 to 44 days). There were no indications for cumulative toxicity. Dose reduction did not result in diminished skin toxicity. Treatment with antihistamines and corticosteroids only minimally alleviated the symptoms. One patient, who experienced a grade 3 rash in the first course, received subsequent cycles of treatment with dexamethasone $3 \mathrm{mg}$ bid from the day before the start of therapy until the day after the last ZD9331 administration in each cycle. In this patient rash was prevented in all further 13 cycles.

Another frequently observed nonhematologic side effect was fever in absence of infection. It was documented in $21 \%$ of the cycles and in 17 out of 42 patients, with a median day of onset of day 5 (range, days 2 to 20) and a median duration of 2 days.

Other types of nonhematologic side effects (nausea, vomiting, mucositis, myalgia/arthralgia, and alopecia) were not severe (Table 3 ). No grade 3 or 4 nausea or vomiting was observed. Grade 1 or 2 nausea and vomiting occurred in $32 \%$ of the cycles but did not have any implications for drug administration. Nine patients experienced grade 1 alopecia and one patient experienced grade 2 alopecia. In $14 \%$ of the cycles, in 12 out of 42 patients, a transient asymptomatic rise in transaminases was observed at all doses with a median day of onset of day 5. Four patients had grade 3 or 4 bilirubinemia in conjunction with elevated levels of liver transaminases; in two of them, the toxicity was considered to be drug related. The grade 3 or 4 bilirubinemia occurred in all but one patient at doses $\geq 20 \mathrm{mg} / \mathrm{d}$ and during the third or subsequent cycles. There was no association between dosage and regimen of ZD9331 and the incidence of hepatic and renal abnormalities.

Hematologic toxicity was mild and mostly limited to grade 1 or 2 neutropenia and thrombocytopenia. Grade 3 or 4 neutropenia and thrombocytopenia were only observed in six patients (Table 3). No treatment-related deaths were reported.

\section{Responses}

One partial response was observed, lasting 30 weeks, in a patient with gastric cancer. Eighteen patients experienced stabilization of their disease (10 patients with colorectal carcinoma, two patients with non-small-cell lung cancer, and one patient each with adenocarcinoma of unknown primary, carcinoma of the stomach, carcinoma of the bladder, pancreatic carcinoma, renal carcinoma, and carcinoma of the cervix) for a median duration of 18 weeks (range, 11 to $52+$ weeks).

\section{Pharmacokinetics and Pharmacodynamics}

Full kinetic data were obtained from all 42 patients after the administration of ZD9331. The plasma concentrationtime profiles of ZD9331 were similar for all patients studied, with a representative profile shown in Fig 3. ZD9331 pharmacokinetics were characterized by rapid absorption after oral dosing, with peak plasma levels occurring at approximately 1 to 2 hours after dosing. The mean estimated pharmacokinetic data for ZD9331 are summarized in Table 4 as a function of the dose. Dose escalation of oral ZD9331 from 2.5 to $20 \mathrm{mg} / \mathrm{d}$ resulted in a linear increase in AUC. Increasing the dose over $20 \mathrm{mg}$ per administration did not result in a further rise of the AUC. On 


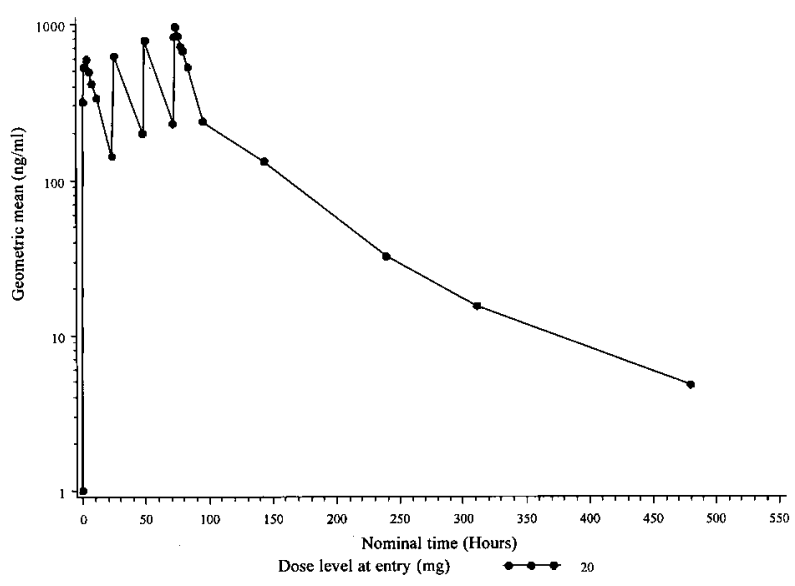

Fig 3. Geometric mean plasma concentration-time profile of ZD9331 measured in the first treatment course after oral administration of ZD9331 at a dose level of $20 \mathrm{mg}$ in a daily-times-five schedule ( $\mathbf{n}=12$ ).

the basis of this observation, alternative regimens were studied with prolonged administration of ZD9331 or multiple dosing per day. The plasma drug exposures per cycle $\left(\mathrm{AUC}_{0-504}\right)$ for the 20-mg od 5-day regimen, 10-mg od 10-day regimen, and 10-mg bid 5-day regimen, all of which resulted in a total dose per cycle of $100 \mathrm{mg}$, were comparable. In addition, similar exposure was observed for the 20-mg od 7-day regimen (Table 4).

Substantial interpatient kinetic variability was apparent, with coefficients of variation in AUC values up to $50 \%$. In one patient, treated with dexamethasone to prevent skin toxicity, additional pharmacokinetic sampling was performed in the fourth cycle. The AUC of ZD9331 showed an increase of approximately $40 \%$ compared with cycle 1 (data not shown). It is not possible to conclude whether these data represent a real increase or are a reflection of the intrapatient variability in pharmacokinetics. However, the maximal concentration and serum half-life of ZD9331 was comparable between the two cycles. Elimination of ZD9331 from the central plasma compartment was characterized by decay in an apparent biexponential manner, based on conventional compartmental modeling using weighted least-squares analysis with a weighting factor of $1 / Y$. The estimated elimination half-life was relatively constant in all subjects (ie, 3.5 days) and was not dependent on the dose of ZD9331. The long half-life resulted in accumulation of ZD9331, such that exposure was two-fold higher by the last day of dosing (data not shown).

The apparent oral clearance of ZD9331 based on the first 24-hour sampling time period was independent of the administered dose $(P=.080)$. Similarly, the $\mathrm{AUC}_{0-24}$ was linearly related to the administered ZD9331 dose (Spearman's rho $=0.53$ ). The apparent volume of distribution ranged from 62.3 to $256.5 \mathrm{~L}$, which suggests significant distribution of ZD9331 into the extravascular tissues. Using linear regression analysis, we found that ZD9331 oral clearance was not significantly related to patient bodysurface area $\left(P=.56 ; r^{2}=.0087\right)$, consistent with previous observations. ${ }^{16}$ In a univariate regression analysis, oral clearance was also not related to patient age $(P=.914$; $\left.r^{2}=.0003\right)$, and no differences were observed between male and female patients $(P=.085$, two-tailed unpaired Student's $t$ test). In an attempt to correlate variability in oral clearance of ZD9331 with renal and hepatic function of individual patients, several additional analyses were conducted using clearance as the independent variable. These revealed that $\mathrm{AUC}_{0-504}$ in the entire population was strongly related to serum creatinine $(P=.0001$; $\left.r^{2}=0.45\right)$ and also, albeit weakly, to baseline bilirubin levels $\left(P=.038 ; r^{2}=.10\right)$ but not to AST $\left(P=.11 ; r^{2}\right.$ $=.062), \operatorname{ALT}\left(P=.15 ; r^{2}=.052\right)$, or alkaline phosphatase $\left(P=.97 ; r^{2}=.00001\right)$.

Table 4. Summary of Pharmacokinetics of Oral ZD9331

\begin{tabular}{|c|c|c|c|c|c|c|c|}
\hline Dose $(\mathrm{mg}) \times$ Days & $\begin{array}{l}\text { Total Dose per Cycle } \\
\text { (mg) }\end{array}$ & $\begin{array}{l}\text { No. of } \\
\text { Patients }\end{array}$ & $C_{\max }(\mathrm{ng} / \mathrm{mL})$ & $\begin{array}{l}\text { AUC }_{0-24 h o u r s} \\
(\mu \mathrm{g} \cdot \mathrm{h} / \mathrm{mL})\end{array}$ & $\begin{array}{c}\mathrm{AUC}_{0-\infty} \\
(\mu \mathrm{g} \cdot \mathrm{h} / \mathrm{mL})\end{array}$ & $C L / f(L / h)$ & $t_{1 / 2}$ (hours) \\
\hline $2.5 \mathrm{od} \times 5$ & 12.5 & 2 & $183 \pm 32$ & $1.79 \pm 0.40$ & $17.8 \pm 0.70$ & $1.17 \pm 1.67$ & $61.9 \pm 28.9$ \\
\hline 5 od $\times 5$ & 25 & 1 & 156 & 1.88 & 30.2 & 2.66 & 155 \\
\hline 10 od $\times 5$ & 50 & 1 & 614 & 7.61 & 103 & 1.31 & 104 \\
\hline 20 od $\times 5$ & 100 & 12 & $686 \pm 238$ & $8.22 \pm 3.40$ & $75.9 \pm 34.7$ & $2.71 \pm 2.57$ & $86.4 \pm 40.3$ \\
\hline 30 od $\times 5$ & 150 & 3 & $846 \pm 208$ & $12.3 \pm 2.74$ & $101 \pm 13.2$ & $2.49 \pm 0.581$ & $93.4 \pm 57.3$ \\
\hline 40 od $\times 5$ & 200 & 6 & $897 \pm 265$ & $10.9 \pm 4.45$ & $108 \pm 38.6$ & $4.08 \pm 1.82$ & $96.0 \pm 29.8$ \\
\hline 10 bid $\times 5$ & 100 & 6 & $797 \pm 192$ & $10.9 \pm 1.84$ & $108 \pm 31.0$ & $1.86 \pm 0.332$ & $93.4 \pm 43.7$ \\
\hline 10 od $\times 10$ & 100 & 6 & $485 \pm 103$ & $5.24 \pm 1.65$ & $124 \pm 33.3$ & $2.01 \pm 0.634$ & $53.6 \pm 20.2$ \\
\hline 20 od $\times 7$ & 140 & 5 & $721 \pm 234$ & $8.21 \pm 1.84$ & $135 \pm 25.0$ & $2.53 \pm 0.819$ & $81.9 \pm 16.9$ \\
\hline
\end{tabular}

NOTE. Kinetic values are mean values \pm SD.

Abbreviations: $C_{\max }$ peak plasma level; $A U C$, area under the plasma concentration-time curve; $C L / f$, apparent total body clearance; $t_{1 / 2}$, terminal elimination half-life. 

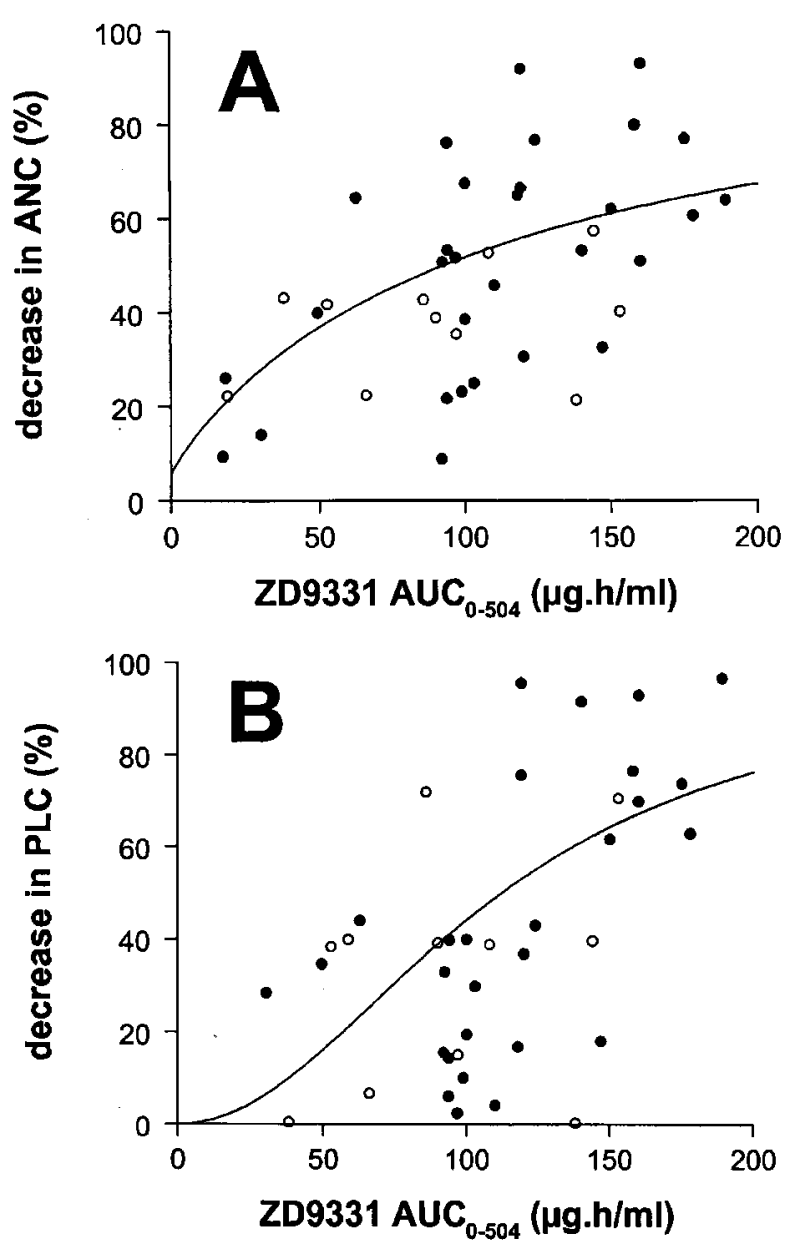

Fig 4. Relationships between $\mathrm{AUC}_{0-504}$ of ZD9331 and hematologic pharmacodynamics, expressed as percentage decrease in absolute neutrophil count (A) and platelet count (B). The open symbols represent data from patients treated at the $20-\mathrm{mg}$ dose level in a daily-times-five schedule.

Correlations were also attempted between pharmacokinetic values based on $\mathrm{AUC}_{0-504}$ values and the experienced hematologic and skin toxicity attained after the first cycle. Hematologic pharmacodynamic analysis revealed no significant relationship between the percentage decrease in WBC $(P<.10)$, although $\mathrm{AUC}_{0-504}$ was related to both the percentage decrease in absolute neutrophil count $(P=$ $\left..0004 ; r^{2}=.55\right)$ and platelet count $\left(P=.0001 ; r^{2}=.63\right)$ using a sigmoidal maximum-effect model (Fig 4). For skin toxicity, although the patients with the highest exposure were linked to grade 3 or 4 toxicity, there did not seem to be a direct relationship between AUC values and toxicity. A similar relationship could be established between the absolute dose of ZD9331 administered and the percentage decrease in absolute neutrophil count $\left(P=.008 ; r^{2}=.401\right)$

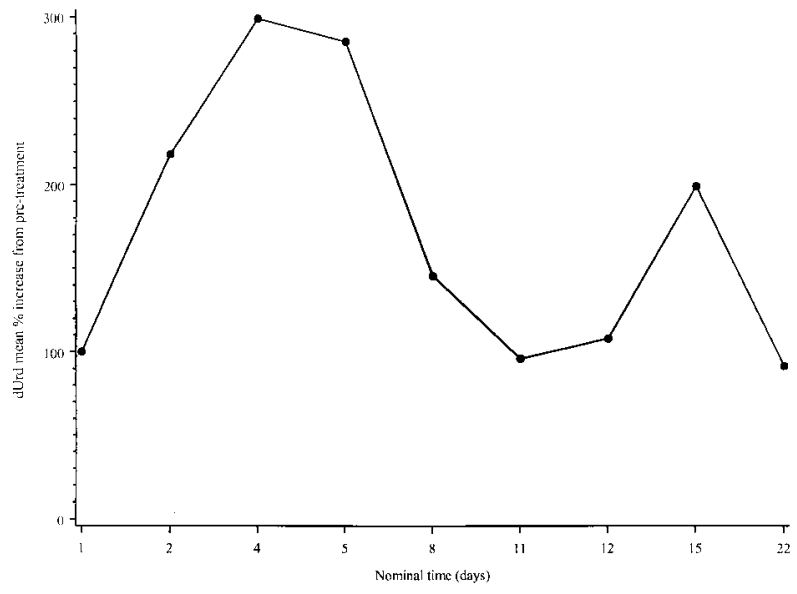

Fig 5. Mean percentage increase in plasma dUrd compared with pretreatment values during cycle 1 in five patients, after oral administration of ZD9331 at $20 \mathrm{mg}$ od for 5 days.

and platelet count $\left(P=.031 ; r^{2}=.329\right)$. In those patients from whom blood samples were collected for analysis of plasma dUrd, increased levels of plasma dUrd were demonstrated in all but one patient. Figure 5 depicts the elevation of plasma dUrd for patients in the 20-mg od 5-day regimen. The maximum rise in plasma dUrd, usually $200 \%$ to $400 \%$ of the pretreatment value, was generally observed on day 2. No correlation between dUrd rise and dose or regimen could be discerned, and in most patients, dUrd levels returned to pretreatment values from 3 days after dosing onward.

\section{DISCUSSION}

The development of orally administered anticancer agents provides a convenient method of prolonged drug administration and the opportunity for cost-effective outpatient therapy. Moreover, patients have a clear preference for oral chemotherapy when equally effective as intravenous administration. ${ }^{23}$ Several TS inhibitors, such as capecitabine and uracil plus tegafur, have shown promising results by oral administration. ${ }^{24,25}$ ZD9331 is a novel TS inhibitor that does not need to be activated by FPGS, thereby circumventing a common form of resistance of the tumor cell to TS inhibition. This, however, also results in a limited intracellular retention time of ZD9331 and might result in the requirement for prolonged dosing schedules in order to optimize drug exposure to inhibit TS and achievement of antitumor efficacy. When prolonged exposure is desirable, oral administration is certainly the most appealing route of administration. The aim of this phase I study was to assess the toxicity profile of oral ZD9331, to determine the 
recommended dose for further study, to assess antitumor efficacy, and to study the pharmacokinetics of the drug.

The DLTs of oral ZD9331 in our study consisted of a combination of skin toxicity and myelosuppression. The skin toxicity observed was a rash, predominantly located on the trunk and extremities. This is in contrast to the hand-foot syndrome, the skin toxicity most often observed during treatment with other TS inhibitors. Furthermore, after the intravenous administration of ZD9331, skin toxicity was also observed, albeit less frequently. ${ }^{15,16,26}$ This difference in frequency of occurrence was not attributed to an altered metabolism of ZD9331 after oral administration, as a recent ${ }^{14} \mathrm{C}$ clinical study has shown that the parent compound is responsible for the majority of circulating activity. ${ }^{27}$ The median day of onset of the skin rash was day 7 , independent of the dosing regimen studied. If a patient experienced skin toxicity in the first cycle, this was predictive for skin toxicity in following cycles. The reappearance of the rash in the following cycles was prevented by pretreatment with dexamethasone. Since at the recommended dose level grade 3 rash only was seen in one patient, prophylactic treatment with corticosteroids for all patients is not recommended.

In 12 out of 42 patients ( $14 \%$ of the cycles), a transient asymptomatic rise in liver transaminases could be observed. This phenomenon is also known from therapy with raltitrexed, during which treatment $13 \%$ of the patients will develop a transient rise in transaminases. ${ }^{28}$ After the intravenous administration of ZD9331, elevated transaminases were observed in 17 of 59 patients. ${ }^{16}$ Other nonhematologic and hematologic side effects were mild. In trials studying the intravenous administration of ZD9331, the DLT consisted of a combination of neutro- and thrombocytopenia. In view of the observed toxicity and the pharmacokinetic data in the present study, indicating that optimal exposure to ZD9331 was achieved with the regimens administering $100 \mathrm{mg}$ of ZD9331 per cycle, $20 \mathrm{mg}$ od for 5 days, repeated every 3 weeks, is recommended for further studies of this regimen.

In the present study, the pharmacokinetics of ZD9331 were linear in the dose range 2.5 to $20 \mathrm{mg}$ od. However, at higher doses, a nonlinear increase in AUC was observed. This phenomenon has also been described after the intravenous administration of the drug, albeit to a lesser extent. ${ }^{16,26}$ Also, multiple daily dosing or extension of the treatment time did not result in an increase in exposure to the drug per cycle. The exposure to ZD9331 per cycle was comparable among the three regimens administering $100 \mathrm{mg}$ per cycle, ie, $20 \mathrm{mg}$ od for 5 days, $10 \mathrm{mg}$ bid for 5 days, and $10 \mathrm{mg}$ od for 10 days. Dose-dependent nonlinearity can be caused by a number of mechanisms. Since the drug is administered orally, absorption might become saturated above a certain dose administered. In that case, however, one would have expected that multiple daily dosing would have improved the exposure to the drug. Oral bioavailability studies of ZD9331 are currently ongoing. A dose-dependent nonlinearity in drug exposure could also be the result of the saturation of reabsorption processes. Excretion of ZD9331 in both the rat and dog was mainly via the bile. In the rat, biliary recirculation of ZD9331 could encompass a saturable active transport system. Enterohepatic circulation of ZD9331 in man has not yet been demonstrated. Saturation of renal tubular reabsorption or nonlinear protein binding may also account for a change in clearance with increasing dose.

ZD9331 was administered at fixed doses during the study. Using linear regression analysis, ZD9331 oral clearance was not significantly related to patient body-surface area, which confirms that the application of dosing per body-surface area would not have optimized dosing.

Inhibition of TS leads to a rise in the level of intracellular dUMP, which is phosphorylated to dUrd. dUrd is effluxed from cells, leading to a rise in plasma dUrd. ${ }^{20,21}$ Thus, plasma dUrd can be used as a surrogate marker for TS inhibition. ${ }^{19,22}$ The limited sampling for the determination of plasma dUrd undertaken during this study confirmed that plasma levels of dUrd increased above pretreatment values for the duration of the drug administration, thereby suggesting that plasma exposure to ZD9331 was sufficient to induce TS inhibition.

In view of the long half-life of ZD9331 and the indication of the existence of TS inhibition even 3 days after drug administration, alternative schedules of drug dosing might provide a better balance between toxicity and efficacy.

The current study has shown that oral ZD9331 has some antitumor activity in a range of tumor types. One partial response was observed in a patient with metastatic gastric cancer, and in another 18 patients, disease stabilization was achieved.

\section{REFERENCES}

1. Carreras CW, Santi DV: The catalytic mechanism and structure of thymidylate synthase. Annu Rev Biochem 64:721-762, 1995

2. Jackman AL, Calvert AH: Folate-based thymidylate synthase inhibitors as anticancer agents. Ann Oncol 6:871-881, 1995
3. Touroutoglou N, Pazdur R: Thymidylate synthase inhibitors. Clin Cancer Res 2:227-243, 1996

4. Takimoto CH: Antifolates in clinical development. Semin Oncol 24:S18-40-S18-51, 1997 (suppl 18) 
5. Jackman AL, Kelland LR, Kimbell R, et al: Mechanisms of acquired resistance to the quinazoline thymidylate synthase inhibitor ZD1694 (Tomudex) in one mouse and three human cell lines. Br J Cancer 71:914-924, 1995

6. Takemura Y, Gibson W, Kimbell R, et al: Cellular pharmacokinetics of ZD1694 in cultured human leukemia cells sensitive, or made resistant, to this drug. J Cancer Res Clin Oncol 122:109-117, 1996

7. Jackman AL, Kimbell R, Brown M, et al: The antitumour activity of ZD9331, a non-polyglutamatable quinazoline thymidylate synthase inhibitor. Adv Exp Med Biol 370:185-188, 1994

8. Takemura Y, Kobayashi H, Gibson W, et al: The influence of drug-exposure conditions on the development of resistance to methotrexate or ZD1694 in cultured human leukemia cells. Int J Cancer 66:29-36, 1996

9. Kobayashi H, Takemura Y, Miyachi H: Molecular characterization of human acute leukemia cell line resistant to ZD9331, a non-polyglutamatable thymidylate synthase inhibitor. Cancer Chemother Pharmacol 42:105-110, 1998

10. Freemantle SJ, Jackman AL, Kelland LR, et al: Molecular characterisation of two cell lines selected for resistance to the folatebased thymidylate synthase inhibitor, ZD1694. Br J Cancer 71:925930, 1995

11. Sirotnak FM, DeGraw JI, Moccio DM, et al: New folate analogues of the 10-deaza-aminopterin series: Basis for structural design and biochemical and pharmacologic properties. Cancer Chemother Pharmacol 12:18-25, 1984

12. Sirotnak FM: Obligate genetic expression in tumor cells of a fetal membrane property mediating "folate" transport: Biological significance and implications for improved therapy of human cancer. Cancer Res 45:3992-4000, 1985

13. Jackman AL, Kimbell R, Aherne GW, et al: Cellular pharmacology and in vivo activity of a new anticancer agent, ZD9331: A water-soluble, non-polyglutamatable, quinazoline-based inhibitor of thymidylate synthase. Clin Cancer Res 3:911-921, 1997

14. Stephens TC, Smith MN, McCloskey ML: ZD9331, a novel non-polyglutamated thymidylate synthase inhibitor: In vivo antitumour efficacy and toxicity to normal murine tissues. Proc Am Assoc Cancer Res 35:305, 1994 (abstr)

15. Diab S, Britten C, Smith R, et al: A phase I pharmacokinetic study of ZD9331, a novel long-acting thymidylate synthase inhibitor on a single dosing every 3 weeks schedule. 10th NCI-EORTC Symp New Drugs Cancer Ther 1998, p 160 (abstr 611)

16. Goh BC, Ratain MJ, Bertucci D, et al: Phase I study of ZD9331 on short daily intravenous bolus infusion for 5 days every 3 weeks with fixed dosing recommendations. J Clin Oncol 19:1476-1484, 2001
17. Plummer R, Rees C, Judson I, et al: Phase I trial of ZD9331 in adult patients with refractory solid malignancies administered by 30-minute infusion on days 1 and 8 with the cycle repeated every 3 weeks. Eur J Cancer 35:S285, 1999 (suppl 4, abstr 1143)

18. Trigo J, Rees C, Beale P, et al: Phase I trial of ZD9331, a non-polyglutamatable thymidylate synthase inhibitor given as a 5-day continuous infusion every 3 weeks. Eur J Cancer 35:S286, 1999 (supp1 4, abstr 1149)

19. Aherne W, Hardcastle A, Kelland L, et al: The measurement of deoxynucleotide (dNTP) pools by radioimmunoassay (RIA). Adv Exp Med Biol 370:801-804, 1994

20. Aherne W, Hardcastle A, Brown S, et al: The effect of ZD9331, a non-polyglutamated quinazoline thymidylate synthase inhibitor, on TTP and dUMP pools. Br J Cancer 71:67, 1995 (suppl 24, abstr 171)

21. Mitchell F, Lynn S, Jackman AL: Modified high-performance liquid chromatography assay for the measurement of 2'-deoxyuridine in human plasma and its application to pharmacodynamic studies of antimetabolite drugs. J Chromatogr B Biomed Sci Appl 744:351-358, 2000

22. Jackman AL, Mitchell F, Lynn S, et al: Plasma 2'deoxyuridine (dUrd) as a surrogate marker of thymidylate synthase (TS) inhibition in patients treated with ZD9331. Proc Am Soc Clin Oncol 18:171a, 1999 (abstr 654)

23. Liu G, Franssen E, Fitch MI, et al: Patient preferences for oral versus intravenous palliative chemotherapy. J Clin Oncol 15:110-115, 1997

24. Budman DR, Meropol NJ, Reigner B, et al: Preliminary studies of a novel oral fluoropyrimidine carbamate: Capecitabine. J Clin Oncol 16:1795-1802, 1998

25. Hoff PM: The tegafur-based dihydropyrimidine dehydrogenase inhibitory fluoropyrimidines, UFT/leucovorin (ORZEL) and S-1: A review of their clinical development and therapeutic potential. Invest New Drugs 18:331-342, 2000

26. Rees C, Beale P, Trigo J, et al: Phase I trial of ZD9331, a non-polyglutamatable thymidylate synthase (TS) inhibitor given as a 5-day continuous infusion. Proc Am Soc Clin Oncol 18:171a, 1999 (abstr 657)

27. Scurr M, Judson I, O’Donnell A, et al: ZD9331: A phase I trial to assess the metabolism, excretion and pharmacokinetics of singledose $260 \mathrm{mg} / \mathrm{m}^{2}\left[{ }^{14} \mathrm{C}\right]-Z D 9331$ infused over 30 minutes in patients with advanced solid tumours. Proc Am Soc Clin Oncol 20:88a, 2001 (abstr 350)

28. Cocconi G, Cunningham D, Van Cutsem E, et al: Open, randomized, multicenter trial of raltitrexed versus fluorouracil plus high-dose leucovorin in patients with advanced colorectal cancer. J Clin Oncol 16:2943-2952, 1998 